European

Thyroid Journal
Eur Thyroid J 2019;8:41-45

DOI: $10.1159 / 000493978$
Received: August 13, 2018

Accepted after revision: September 21, 2018

Published online: November 16, 2018

\title{
Repeat Ultrasonography in the First Years after Therapy with Radioiodine Is Not Necessary in Most Patients with Papillary Thyroid Carcinoma when Postoperative Ultrasonography Is Negative: A Reduction of Costs and False-Positives
}

\author{
Pedro Weslley Rosario Gabriela Franco Mourão Maria Regina Calsolari \\ Santa Casa de Belo Horizonte, Belo Horizonte, Brazil
}

\section{Keywords \\ Papillary thyroid carcinoma - Ultrasound $\cdot$ Thyroglobulin}

\begin{abstract}
Background: Periodic ultrasonography (US) examination is recommended in many patients with papillary thyroid carcinoma (PTC) after treatment with radioactive iodine (RAl), but recurrences are confirmed in few cases. This study determined whether the indication of US in the first years after treatment with RAI can be selective when postoperative US and posttherapy whole-body scanning (RxWBS) ruled out persistent neck disease. Methods: This was a prospective study. Two hundred and fifty-four patients with PTC (242 of intermediate risk) undergoing thyroidectomy and RAI, who had no apparent disease at the time of initial therapy (including negative postoperative US and RxWBS), were studied. The patients were followed up for 5 years after treatment with RAI by annual US. Results: At the end of 5 years, 47 patients (18.5\%) had at least one suspicious US, but neck recurrence was confirmed in only 4 patients ( $1.5 \%$ of all patients and $8.5 \%$ of those with suspicious US). The remaining cases were considered false-positives. US did not reveal disease in the first or second year after treatment with RAI in any pa-
\end{abstract}

tient. In the third, fourth, and fifth year after treatment with $\mathrm{RAl}$, considering only patients with unstimulated $\mathrm{Tg}<1 \mathrm{ng} /$ $\mathrm{mL}$ in these assessments, US revealed disease in $0,1(0.4 \%)$, and $1(0.4 \%)$ patient, respectively. Conclusion: The results suggest that low- or intermediate-risk patients with PTC without persistent disease after thyroidectomy (including negative postoperative US and RxWBS) do not require repeat US examination in the first two years after treatment with RAI. In the following years until the fifth year, US can be restricted to patients with $\mathrm{Tg} \geq 1 \mathrm{ng} / \mathrm{mL}$.

(c) 2018 European Thyroid Association Published by S. Karger AG, Basel

\section{Introduction}

Ultrasonography (US) is a widely available, noninvasive, and sensitive technique for the detection of locoregional disease in patients operated on for papillary thyroid carcinoma (PTC). After ablation or adjuvant therapy with radioactive iodine (RAI), US should be obtained a few months later in all patients as part of the investigation that defines the response to therapy [1-3]. After this first assessment, the American Thyroid Association (ATA)

\section{KARGER}

E-Mail karger@karger.com

www.karger.com/etj (c) 2018 European Thyroid Association

Published by S. Karger AG, Basel
P.W. Rosario, MD

Instituto de Ensino e Pesquisa, Santa Casa de Belo Horizonte

Rua Domingos Vieira, 590, Santa Efigênia

Belo Horizonte, MG 30150-240 (Brazil)

E-Mail pedrowsrosario@gmail.com 
[2] and the European Thyroid Association (ETA) [1] only exclude the need for repeat US in patients (i) of low risk, (ii) with excellent response to therapy, and (iii) with persistently negative unstimulated $\mathrm{Tg}(\mathrm{u}-\mathrm{Tg})$ and anti-Tg antibodies (TgAb). Even in these cases, the recommendation of repeating US at least every 12-24 months within the first 5 years has recently been reiterated [3]. However, US frequently reveals "suspicious" lesions that raise patient concern and required fine-needle aspiration (FNA) but that are not malignant (false-positives) [4]. Furthermore, most patients with PTC (except high-risk patients) will not develop disease after treatment with RAI [2]. Consequently, the detection of a neck recurrence requires US to be performed in many patients as well as several examinations per patient [4], sometimes followed by FNA, resulting in high costs.

In patients with macroscopically complete tumor resection who receive RAI, persistent locoregional disease is more commonly detected by posttherapy whole-body scanning (RxWBS) [5]. Recently, postoperative US (before RAI) has been shown to be a valuable procedure [1$3,6,7]$, which can even detect lymph node metastases not apparent on RxWBS [6-8]. So far, few studies have routinely obtained postoperative US before RAI in a large number of patients $[6,7]$. These studies were retrospective. Since it was already known that the patients would subsequently receive RAI, it is possible that US was less detailed, since the result would not change the decision on RAI administration, or that only lesions that were candidates for reoperation were reported. The time to obtain US after surgery was also not predefined or standardized. Finally, only one study reported the results of US after RAI in patients with negative postoperative US [6].

The objective of this prospective study was to determine whether the indication of US could be selective in the first years after RAI when postoperative US and RxWBS ruled out persistent neck disease after total thyroidectomy.

\section{Patients and Methods}

\section{Study}

This was a prospective study. The study was approved by the local Research Ethics Committee, and the subjects gave informed consent.

\section{Patients}

The patients of this study were treated at our hospital between 2006 and 2014. During this period until today, patients with PTC have been evaluated routinely 3-4 months after surgery by US/ Doppler. For this study, patients submitted to total thyroidectomy who received RAI and who did not have persistent disease according to the criteria in Table 1 were selected. Patients with poorly differentiated carcinoma and extensive extrathyroid invasion (pT4) were excluded. In addition, to avoid underestimating recurrences, patients with classical PTC $\leq 4 \mathrm{~cm}$ restricted to the thyroid and without vascular invasion were not included. Patients with $\mathrm{TgAb}$ were also excluded. Once included in the study, the patients were followed up for 5 years after treatment with RAI as described below.

\section{Ablation with RAI}

The patients were submitted to ablation with RAI after levothyroxine withdrawal for 4 weeks or administration of recombinant human TSH and administration of a low-iodine diet for 10 days prior to the procedure. 30, 50, or $100 \mathrm{mCi}$ was administered. Anterior and posterior whole-body images were obtained 7 days after RAI (RxWBS).

\section{Initial Assessment after Treatment with RAI}

The patients were evaluated 12 months after the administration of RAI by u-Tg and TgAb measurement and US.

\section{Follow-Up}

The patients were followed up by clinical examination, measurement of $\mathrm{u}-\mathrm{Tg}$ and $\mathrm{TgAb}$ at intervals of 6-12 months, and annual US. The time of follow-up was 60 months in all patients.

\section{Imaging Methods}

US was performed with a linear, multifrequency, $14-\mathrm{MHz}$ transducer for morphological analysis and for power Doppler evaluation. The absence of US abnormalities was defined when no lymph nodes with any of the following findings were detected: (i) microcalcification, (ii) cystic degeneration, (iii) peripheral hypervascularization, and (iv) absence of a hilum with a long axis/short axis ratio $<2$ and no vascularized nodule in the thyroid bed $[9,10]$. US/Doppler was performed by a radiologist experienced in thyroid imaging. All suspected lesions apparent on the scans were evaluated by US-guided FNA (cytology and Tg). True-positive US findings were defined based on the results of cytology, FNA-Tg, and histology.

Assays

Chemiluminescent assays were used for the measurement of $\mathrm{Tg}$ (Access Thyroglobulin Assay, Beckman Coulter, Fullerton, CA, USA [functional sensitivity (FS) in our laboratory of $0.1 \mathrm{ng} / \mathrm{ml}$ ]) and TgAb (Immulite 2000, Diagnostic Products Corporation, Los Angeles, CA, USA [reference value of $\leq 40 \mathrm{IU} / \mathrm{mL}$ and FS of $20 \mathrm{IU} /$ $\mathrm{mL}$ ] or ARCHITECT Anti-Tg, Abbott Laboratories, Abbott Park, IL, USA [reference value of $\leq 4.11 \mathrm{IU} / \mathrm{mL}$ and FS of $1 \mathrm{IU} / \mathrm{mL}$ ]).

\section{Results}

Two hundred and fifty-four patients met the selection criteria (Table 1), i.e., they did not have apparent persistent disease, including the absence of postoperative US abnormalities and RxWBS showing no uptake outside the thyroid bed. There were 197 women and 57 men ranging 
Table 1. Definition of "absence of persistent disease"

- Macroscopically complete tumor resection in the neck as reported by the surgeon

- Clinical examination 3 months after surgery without suspicion of metastases

- Surgical margins not compromised

- Neck US/Doppler 3-4 months after surgery without abnormalities (see Patients and Methods)

- RxWBS showing uptake in the thyroid bed $<2 \%$ [11] and no ectopic uptake

US, ultrasonography; RxWBS, posttherapy whole-body scanning.

in age from 16 to 78 years (mean 49 years). According to ATA [2], 242 would be classified as intermediate risk and only 12 as low risk (tumor $>4 \mathrm{~cm}$ ).

One year after treatment with RAI, US was suspicious in 10 patients, but FNA (cytology and measurement of Tg in the needle washout) excluded the recurrence of a tumor, and these cases were, therefore, considered falsepositive US. In the second year, suspicious US with negative FNA was observed in an additional 8 patients. In the third year, lymph node recurrence was detected by US in one patient. This patient had $\mathrm{u}-\mathrm{Tg} \geq 1 \mathrm{ng} / \mathrm{mL}$ at the time of recurrence detection. Suspicious US with negative FNA was observed in an additional 9 patients. In the fourth year, US was suspicious in 10 further patients, but recurrence was confirmed in only two. One of the patients with recurrence detected by US had $\mathrm{u}-\mathrm{Tg}<1 \mathrm{ng} / \mathrm{mL}$ at diagnosis. Finally, in the fifth year after treatment with RAI, US revealed suspicious lesions in 9 further patients but recurrence was confirmed in only one. This patient had $\mathrm{u}-\mathrm{Tg}<1 \mathrm{ng} / \mathrm{mL}$ at the time of US.

At the end of 5 years, 47 patients (18.5\%) had at least one suspicious US, but neck recurrence was confirmed in only 4 (1.5\% of all patients and $8.5 \%$ of those with suspicious US). The remaining cases were considered falsepositives. Thus, in the absence of persistent neck disease (including no abnormal postoperative US findings and negative RxWBS), US did not reveal structural disease in the first or second year after treatment with RAI in any patient. In the third, fourth, and fifth year after treatment with RAI, considering only patients with $\mathrm{u}-\mathrm{Tg}<1 \mathrm{ng} / \mathrm{mL}$ in these assessments as has been proposed recently [4], US revealed disease in $0,1(0.4 \%)$, and $1(0.4 \%)$ patient, respectively. The two patients with recurrence on US and $\mathrm{u}-\mathrm{Tg}<1 \mathrm{ng} / \mathrm{mL}$ had detectable concentrations of this marker; thus, none of the patients with undetectable $\mathrm{Tg}$

Postoperative Ultrasonography and $\mathrm{TgAb}$ after treatment with RAI exhibited recurrence on US.

To avoid overestimation of the frequency of false-positive results, for the 47 patients who had at least one suspicious US, we considered the number of scans obtained until the first suspicious US. Thus, 1,176 scans were obtained; of these, 1,129 (96\%) did not reveal suspicious lesions, only $4(0.35 \%)$ resulted in the confirmation of recurrence, and 43 (3.65\%) were considered false-positives. It should be noted that, over the period of 5 years, only two neck recurrences were detected by other imaging methods in the absence of corresponding lesions on US.

\section{Discussion}

This study evaluated patient with PTC since, different from follicular carcinoma, recurrences are mainly cervical in this histological type, and US plays, therefore, a more important role. Patients submitted to lobectomy were not included since periodic US seems to be essential for the follow-up of these patients due to the limitations of $\operatorname{Tg}$ and $\operatorname{TgAb}[1,12,13]$ and due to the need for evaluating not only lymph node metastases but also the remnant lobe [1]. Easily available information was used to define "the absence of persistent disease" after thyroidectomy. In fact, RxWBS has always been recommended after RAI therapy [2] and postoperative US (before RAI) is currently also indicated $[1-3,6,7]$. Finally, we restricted follow-up to the first 5 years after treatment with RAI, since most recurrences occur during this period $[1,14$, $15]$ and periodic US examination is more commonly recommended during these years $[1,3]$.

To the best of our knowledge, there is only one previous study in which postoperative US was obtained routinely in a significant number of patients; in this study, $16 / 468$ patients $(3.4 \%)$ with negative US and RxWBS showing no ectopic cervical uptake had recurrences on US scans obtained after treatment with RAI [6]. However, that study was retrospective, with the limitations inherent in its design, a considerable number of patients were high risk, and serum Tg at recurrence detection was not reported. In another recent study that proposes to restrict US to patients with $\mathrm{u}-\mathrm{Tg} \geq 1 \mathrm{ng} / \mathrm{mL}$ after treatment with RAI, this imaging method revealed disease in only $2.1 \%$ of all scans obtained in patients with $\mathrm{u}-\mathrm{Tg}<1 \mathrm{ng} / \mathrm{mL}$ [4]. That study was also retrospective, and postoperative US was not obtained; thus, patients with US abnormalities in this imaging method were not excluded as was done in the present study. 
In the absence of an apparent tumor in the neck after thyroidectomy, including US and RxWBS, repeat US examination in the first year after RAI, as recommended by current guidelines [1-3], did not show disease in any of the 254 patients. The same was observed in the second year. In the subsequent three years, if US were restricted to patients with u-Tg $\geq 1 \mathrm{ng} / \mathrm{mL}$ [4], 95\% of the patients could be exempted from this examination, and the risk of tumor recurrence in the neck going undetected due to the omission of US would be only $0.4 \%$ in these patients. We attributed these results to the following facts. First, US was performed by an experienced professional, US was not obtained immediately after surgery, reducing the risk of artifacts that could compromise appropriate evaluation of the neck $[1,6$, 8], and we adopted a criterion to define "suspicious" US. As previously demonstrated by us, this criterion is highly sensitive [10]. We believe that this care can be applied in clinical practice without major difficulty. Second, in addition to US, RxWBS was also used to rule out persistent disease, and the application of this method after treatment with RAI is a well-established recommendation [2]. It should be noted that we did not perform SPECT/CT, as currently preferred $[2,16]$, which could theoretically increase sensitivity and further improve the negative predictive value of initial assessment. We recognize that the results cannot be applied to high-risk patients. In contrast, the results can be extrapolated to low-risk patients submitted to total thyroidectomy without RAI. In this case, it is possible that the $\mathrm{u}-\mathrm{Tg}$ cut-off excluding repeat US in these patients is $>1 \mathrm{ng} / \mathrm{mL}$ because of the presence of thyroid remnant.

As reported in other studies [4], we demonstrate that US detected many more false-positives than true-positive recurrences. Among patients with at least one "suspi- cious" US, the disease was confirmed in only $8.5 \%$. Considering only US scans performed at the currently recommended times after treatment with RAI, i.e., in the first year and repeated in the third and fifth year [1-3], more than 750 scans were obtained for the detection of 4 recurrences, a fact that renders the detection of one recurrence expensive at least in these patients.

In conclusion, the results suggest that low- or intermediate-risk patients with PTC without persistent disease after total thyroidectomy (including postoperative US and RxWBS) do not require repeat US examination in the first two years after treatment with RAI. In the following years until the fifth year, this imaging method can be restricted to patients with $\mathrm{u}-\mathrm{Tg} \geq 1 \mathrm{ng} / \mathrm{mL}$ [4] and would not be necessary in patients with undetectable $\mathrm{Tg}$ and TgAb.

\section{Statement of Ethics}

The study was approved by the Research Ethics Committee of our institution, and the subjects gave informed consent.

\section{Disclosure Statement}

The authors declare that there is no conflict of interest that could be perceived as prejudicing the impartiality of the research reported.

\section{Funding Sources}

This work was supported by the National Council for Scientific and Technological Development (CNPq).

\section{References}

1 Leenhardt L, Erdogan MF, Hegedus L, Mandel SJ, Paschke R, Rago T, et al. 2013 European thyroid association guidelines for cervical ultrasound scan and ultrasound-guided techniques in the postoperative management of patients with thyroid cancer. Eur Thyroid J. 2013 Sep;2(3):147-59.

2 Haugen BR, Alexander EK, Bible KC, Doherty GM, Mandel SJ, Nikiforov YE, et al. 2015 American Thyroid Association Management Guidelines for Adult Patients with Thyroid Nodules and Differentiated Thyroid Cancer: The American Thyroid Association Guidelines Task Force on Thyroid Nodules and Differentiated Thyroid Cancer. Thyroid. 2016 Jan;26(1):1-133.
3 Pacini F, Basolo F, Bellantone R, Boni G, Cannizzaro MA, De Palma M, et al. Italian consensus on diagnosis and treatment of differentiated thyroid cancer: joint statements of six Italian societies. J Endocrinol Invest. 2018 Jul;41(7):849-76.

4 Verburg FA, Mäder U, Giovanella L, Luster M, Reiners C. Low or Undetectable Basal Thyroglobulin Levels Obviate the Need for Neck Ultrasound in Differentiated Thyroid Cancer Patients After Total Thyroidectomy and 131I Ablation. Thyroid. 2018 Jun;28(6): $722-8$.
5 Leboulleux S, Rubino C, Baudin E, Caillou B, Hartl DM, Bidart JM, et al. Prognostic factors for persistent or recurrent disease of papillary thyroid carcinoma with neck lymph node metastases and/or tumor extension beyond the thyroid capsule at initial diagnosis. J Clin Endocrinol Metab. 2005 Oct;90(10):5723-9.

6 Lepoutre-Lussey C, Maddah D, Golmard JL, Russ G, Tissier F, Trésallet C, et al. Post-operative neck ultrasound and risk stratification in differentiated thyroid cancer patients with initial lymph node involvement. Eur J Endocrinol. 2014 Jun;170(6):837-46. 
7 Matrone A, Gambale C, Piaggi P, Viola D, Giani C, Agate L, et al. Postoperative Thyroglobulin and Neck Ultrasound in the Risk Restratification and Decision to Perform 131I Ablation. J Clin Endocrinol Metab. 2017 Mar; 102(3):893-902.

8 de Rosário PW, Guimarães VC, Maia FF, Fagundes TA, Purisch S, Padrao EL, et al. Thyroglobulin before ablation and correlation with posttreatment scanning. Laryngoscope. $2005 \mathrm{Feb} ; 115(2): 264-7$.

9 Rosário PW, de Faria S, Bicalho L, Alves MF, Borges MA, Purisch S, et al. Ultrasonographic differentiation between metastatic and benign lymph nodes in patients with papillary thyroid carcinoma. J Ultrasound Med. 2005 Oct;24(10):1385-9.
10 Rosário PW, Tavares WC, Borges MA, Santos JB, Calsolari MR. Ultrasonographic differentiation of cervical lymph nodes in patients with papillary thyroid carcinoma after thyroidectomy and radioiodine ablation: a prospective study. Endocr Pract. 2014 Apr;20(4): 293-8.

11 Cailleux AF, Baudin E, Travagli JP, Ricard M, Schlumberger M. Is diagnostic iodine-131 scanning useful after total thyroid ablation for differentiated thyroid cancer? J Clin Endocrinol Metab. 2000 Jan;85(1):175-8.

12 Cho JW, Lee YM, Lee YH, Hong SJ, Yoon JH. Dynamic risk stratification system in post-lobectomy low-risk and intermediate-risk papillary thyroid carcinoma patients. Clin Endocrinol (Oxf). 2018 Jul;89(1):100-9.
13 Park S, Jeon MJ, Oh HS, Lee YM, Sung TY, Han M, et al. Changes in Serum Thyroglobulin Levels After Lobectomy in Patients with Low-Risk Papillary Thyroid Cancer. Thyroid. 2018 Aug;28(8):997-1003.

14 Durante C, Montesano T, Torlontano M, At$\operatorname{tard}$ M, Monzani F, Tumino S, et al.; PTC Study Group. Papillary thyroid cancer: time course of recurrences during postsurgery surveillance. J Clin Endocrinol Metab. 2013 Feb; 98(2):636-42.

15 Kim H, Kim TH, Choe JH, Kim JH, Kim JS Oh YL, et al. Patterns of Initial Recurrence in Completely Resected Papillary Thyroid Carcinoma. Thyroid. 2017 Jul;27(7):908-14.

16 Hassan FU, Mohan HK. Clinical Utility of SPECT/CT Imaging Post-Radioiodine Therapy: Does It Enhance Patient Management in Thyroid Cancer? Eur Thyroid J. 2015 Dec; 4(4):239-45. 